\title{
Value of cord blood Treg population properties and function-associated characteristics for predicting allergy development in childhood
}

\author{
VIKTOR ČERNÝ', PETRA PETRÁSKOVÁl, OLGA NOVOTNÁ', KRISTÝNA BORÁKOVÁ2, \\ LUDMILA PROKEŠOVÁ ${ }^{1}$ LIBUŠE KOLÁR̆OVÁ', JIŘI HRDÝ ${ }^{1}$
}

${ }^{1}$ Institute of Immunology and Microbiology, First Faculty of Medicine, Charles University and General University Hospital in Prague, Czech Republic ${ }^{2}$ Institute for the Care of Mother and Child, Prague, Czech Republic

\begin{abstract}
Allergic diseases represent some of the most common immunological disorders with high clinical and economic impact. Despite intensive research, there are still few universally accepted and reliable biomarkers capable of predicting their development at an early age.

There is therefore a pressing need for identification of potential predictive factors and validation of their prognostic value by correlating them with allergy development.

Dysbalance of the branches of immune response, most often excessive Th2 polarization, is the principal cause of allergic diseases. Regulatory T cells (Treg) are a crucial population for the timely establishment of physiological immune polarization and induction and maintenance of tolerance against environmental antigens. This makes them a potentially promising candidate for an early marker predicting allergy development.

In our study, we analysed samples of cord blood of children of allergic mothers and children of healthy mothers by flow cytometry and retrospectively correlated the data with clinical allergy status of the children at the age of 6 to 10 years.

Studied parameters included cord blood Treg population proportions and functional properties intracellular presence of IL-10 and TGF- $\beta$, MFI of FoxP3. We observed higher percentage of Tregs in cord blood of children who did not develop allergy compared with allergic children. Further, we found higher numbers of IL-10+ Tregs in cord blood of healthy children of healthy mothers than in cord blood of children of allergic mothers and decreased TGF- $\beta+$ cord blood Tregs in the group of allergic children of allergic mothers compared to all other groups.
\end{abstract}

Key words: cord blood, Treg, regulatory T cells, allergy, TGF- $\beta, I L-10$.

(Centr Eur J Immunol 2020; 45 (4): 393-402)

\section{Introduction}

Allergy remains one of the most common medical conditions, with significant clinical and economical relevance. Allergic diseases represent a highly variable, multifactorial group of disorders characterized by immune dysregulation and failure to develop tolerance towards innocuous environmental antigens. This underlying dysbalance has its roots as early as in the perinatal period. Prenatally, the foetus is maintained under Th2 bias to prevent unwanted reactivity towards antigenically foreign maternal determinants [1]. During the course of pregnancy, immunosuppressive environment supporting regulatory subpopulations is present in uterus under hormonal control [2]. Postnatally, new balance among the branches of immune system needs to be established - persisting Th2 predominance promotes sensitization and allergy development, while Th1 and Th17 branches play crucial roles in anti-infectious immunity but unchecked can cause autoimmune or inflammatory diseases [3].

Generally, neonatal immune system is immature. Proper development of immune responses together with setting tolerance to food antigens and compounds of microbiota is therefore necessary. This fine tuning of immunity in the early postnatal period is highly regulated and involves both intrinsic and extrinsic factors, chiefly exposure to microbial stimuli [4], consistent with the generally accepted hygiene hypothesis [5]. Intrinsically, regulatory $\mathrm{T}$ cells

Correspondence: Jiří Hrdý, Institute of Immunology and Microbiology, First Faculty of Medicine, Charles University and General University Hospital in Prague, Czech Republic, e-mail: jiri.hrdy@1f1.cuni.cz Submitted: 22.07.2019; Accepted: 20.11.2019 
(Tregs) play a key role. This crucial immunomodulatory population of Th lymphocytes serves as master regulators capable of finely tuning balance among the other branches of immune response, maintaining peripheral tolerance and suppressing pathological immune reactions, including Th2-based allergy [3].

Human Tregs are commonly described as $\mathrm{CD} 4{ }^{+} \mathrm{CD} 25^{\text {high- }}$ $\mathrm{CD} 127^{\text {low }}$ population, further characterized by high dependence on IL-2, expression of lineage-specific transcription factor FoxP3 and demethylation of specific sequences within foxp 3 promoter region (Treg-specific demethylated region - TSDR) [6, 7]. The functional relevance of these cells is evidenced by severe autoimmunity as well as allergy accompanying deficiency or impaired function of these cells $[8,9]$. Tregs and their various subpopulations are thus indispensable for controlling allergic diseases both physiologically $[3,10]$ and in the context of specific allergen immunotherapy [11].

Regulatory $\mathrm{T}$ cells utilize numerous mechanisms for their suppressive and immunomodulatory functions, both contact-dependent (presence of cell surface molecules like e.g. CTLA-4, PD-1, LAG3, FasL) and remote (secretion of regulatory cytokines IL-10, TGF- $\beta$ and IL-35) $[12,13]$. Assessment of such Treg-associated markers is commonly used to indirectly assess functional capacity of Tregs, both on the level of individual cells (measured via flow cytometry, e.g. presence of surface or intracellular markers, median of fluorescence intensity [MFI] of FoxP3 [14]) and systemically (e.g. levels of regulatory cytokines in serum measured by ELISA $[15,16])$.

Numerous subpopulations of Tregs were identified by flow cytometry, based upon differential expression of various surface and/or intracellular markers, as well as different biological functions. Gating strategies, sample preparation (particularly antibody clone selection), genetic background, clinical and environmental context and other factors can significantly influence Tregs, introducing discrepancies into published results. Therefore, it is vital to strive for consistency and to carefully consider the epidemiological and methodical aspects when interpreting studies published by different groups [17].

Despite marked progress in understanding the early processes involved in sensitization and allergy development, there is still lack of definite consensus regarding reliable early predictors of an individual's increased risk of allergy. Numerous markers have been analysed in cord blood, including cord blood IgE levels [18], levels of cytokines including IL-10 and TGF- $\beta$ in cord blood [15] and neonatal peripheral blood [19] plasma, as well as reactivity of cord blood cells to various stimuli, with limited conclusiveness when taken together [16, 20-23]. So far, the allergy status of the mother remains the strongest unambiguously accepted predictive factor [23].

Several studies have correlated population characteristics and functional properties of Tregs with atopic diseases in small infants [16, 24-27]. In our own work published by Hrdý in 2012 [17], we reported lower presence of intracellular markers associated with Treg function (IL-10, TGF- $\beta$, MFI of FoxP3) in Tregs from cord blood of children at higher risk of allergy development (based on maternal allergy status). Furthermore, we observed a slightly larger population of Tregs in cord blood of children of allergic mothers, possibly due to compensatory expansion of the population. We concluded by stating the importance of utilizing functional studies and above all the correlation of findings obtained from cord blood samples with the actual development of allergy in later life. In the current study, we perform retrospective analysis of a subset of data from the 2012 report, stratifying the previous findings according to the clinical allergy status of the children at the age of 6 to 10 years.

\section{Material and methods}

\section{Subjects}

The original study included a total of 153 healthy and allergic mothers with physiological pregnancies and children delivered vaginally at full term in the Institute for the Care of Mother and Child in Prague, Czech Republic [17]. The subjects were divided into two groups according to maternal allergic status, based on clinical manifestation of allergy persisting for over two years; monitoring by an allergist; positive skin prick tests and/or specific IgE antibodies; and anti-allergic treatment administered before pregnancy. The study including the current follow-up was approved by the Ethical Committee of the Institute for the Care of Mother and Child (Prague, Czech Republic) and carried out with the signed written informed consent of the mothers.

For the current follow-up study, the families originally included were contacted with a questionnaire in order to collect clinical data regarding manifestation of allergy in the children at the age of 6-10 years, confirmed by clinical examination by an allergist. A total of 39 responding subjects were included in the analysis, with the subjects subdivided into four groups based on combination of the allergy status of the mothers and the children themselves: allergic children of allergic mothers (A/A; $n=8)$, healthy children of allergic mothers $(\mathrm{H} / \mathrm{A} ; n=12)$, allergic children of healthy mothers $(\mathrm{A} / \mathrm{H} ; n=7)$ and healthy children of healthy mothers $(\mathrm{H} / \mathrm{H} ; n=12)$.

\section{Cord blood samples}

Between 10 and $20 \mathrm{ml}$ of umbilical cord blood were collected into sterile heparinised tubes for analysis of Treg population characteristics as well as intracellular presence of transcription factor FoxP3 and regulatory cytokines IL-10 and TGF- $\beta$ by flow cytometry.

\section{Flow cytometry}

Whole blood samples were prepared and stained for flow cytometry analyses as described previously [17]. 
Briefly, samples of whole blood were stained for cell surface markers using the following antibodies: CD4-FITC, clone RPA-T4, cat. no. 555346; CD25- PE-Cy7, clone M-A251, cat. no. 557741; and CD127-Alexa 647, clone HIL-7R-M21, cat. no. 558598, all from Becton Dickinson (Franklin Lakes, NJ, USA). For intracellular staining, following antibodies were used: FoxP3-PE (clone PCH101, cat. no. 12-4776-41A; eBioscience, San Diego, CA, USA); IL-10-PE (clone JES3-19F1, cat. no. 506804) and TGF- $\beta$-PerCP-Cy5.5 (clone BG/hLAP, cat. no. 341803; both from BioLegend).

Proportion of Tregs and percentage of IL-10 and TGF- $\beta$ positive Tregs were determined as described previously [17]. In the current study, data were re-analysed to obtain proportion of Tregs characterised as $\mathrm{CD}^{+-}$ $\mathrm{FoxP}^{+}$using gating strategy shown in the Supplementary Figure 1. Briefly, lymphocytes were gated based on forward-scatter (FSC) and side-scatter (SSC) characteristics, followed by doublets exclusion based on FSC-A $\times$ FSC-H. $\mathrm{CD}^{+} \mathrm{T}$ cells were identified and Tregs then gated from this population as $\mathrm{T}$ cell subset positive for FoxP3.

\section{Data acquisition and statistics}

Flow cytometry data were acquired using BD FACSCanto II flow cytometer using BD FACS Diva version
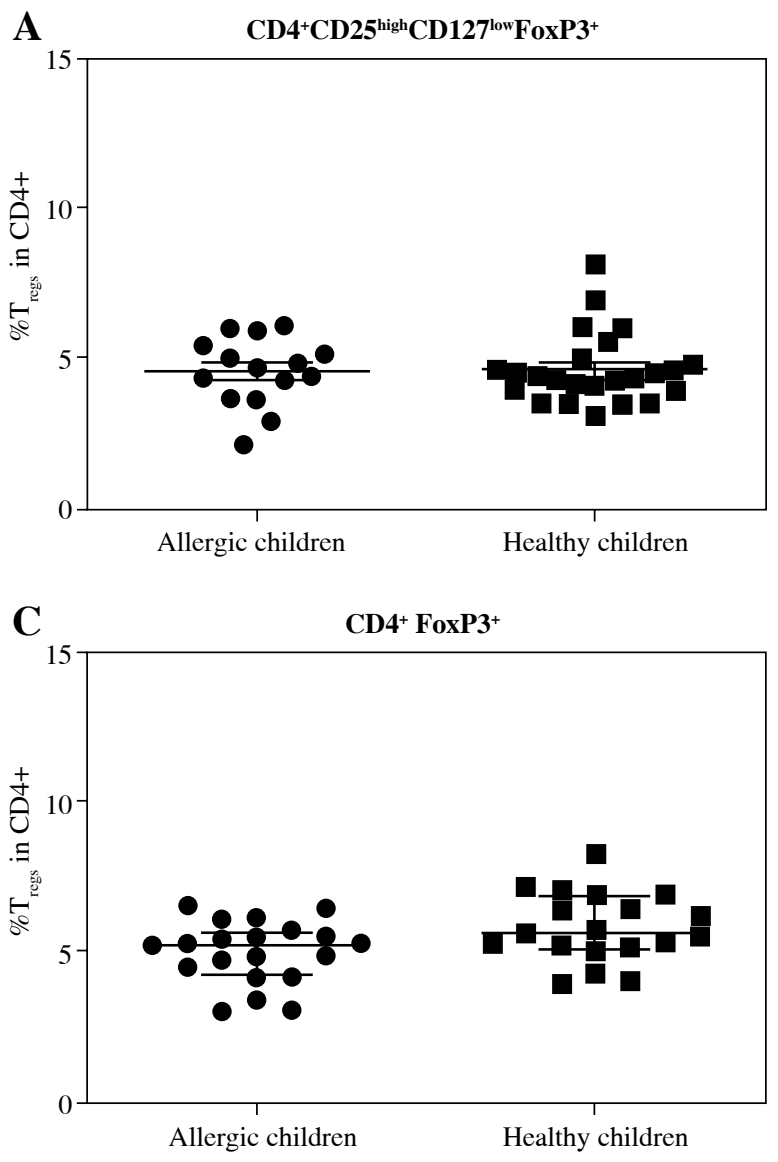

6.1.2 software (Becton Dickinson) and analysed using FlowJo 7.2.2 (TreeStar. Ashland, OR, USA). Statistical and graphical analyses were performed using GraphPad Prism 6.0 (Graph Pad Software, La Jolla, CA, USA). Differences between the groups were compared using unpaired Student's $t$-test in the case of normally distributed data (Treg ratios, MFI of FoxP3) and Mann-Whitney non-parametric test for the proportions of IL- $10^{+}$Tregs and TGF- $\beta^{+}$Tregs.

\section{ELISA}

Concentrations of regulatory cytokines IL-10 and TGF- $\beta$ in the sera of cord blood were quantified by an enzyme-linked immunosorbent assay (ELISA) as described previously [15]. Briefly, primary (MAB 240) and secondary biotinylated (BAF 240) antibodies and recombinant protein (240-IL) for TGF- $\beta$ and Detection kit for IL-10 (DY217-B) were purchased from R\&D Systems. Concentrations of cytokines were calculated from calibration curves and expressed in $\mathrm{pg} / \mathrm{ml}$ using software KIM (Schoeller Instruments).

\section{Results}

The original study analysed immunological characteristics of Tregs from cord blood in the context of expected risk of allergy development, based on maternal allergy sta-

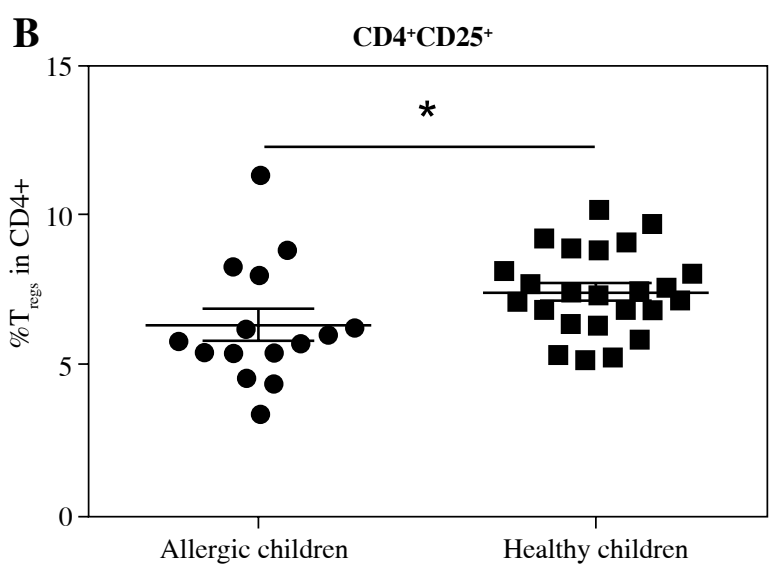

Fig. 1. Proportions of regulatory T cells (Tregs) in cord blood evaluated according to the children's allergy status. Samples of cord blood of allergic children $(n=15)$ and healthy children $(n=24)$ were stained and analysed by flow cytometry. A) Four-color cytometry analysis of CD4 ${ }^{+} \mathrm{CD} 25^{\text {high }} \mathrm{CD} 127^{\text {low }} \mathrm{FoxP} 3{ }^{+}$Tregs; B) Two-color cytometry analysis of $\mathrm{CD} 4{ }^{+} \mathrm{CD} 25^{+}$cells; $\mathbf{C}$ ) Two-color cytometry analysis of $\mathrm{CD}^{+}{ }^{+} \mathrm{FoxP} 3^{+}$Tregs; ${ }^{*} p \leq 0.05$ 
tus [17]. Current follow-up allowed us to correlate the data with actual allergy status of the children, an aspect critically important for considering proportion and functional characteristics of Tregs as possible predictive markers.

The proportion of Tregs, MFI of FoxP3 and intracellular presence of regulatory cytokines (IL-10 and TGF- $\beta$ ) were compared among the healthy and allergic children of healthy mothers and allergic mothers, respectively.

\section{Treg ratio}

When we used the gating strategy described earlier [17] and Tregs were considered as $\mathrm{CD} 4^{+} \mathrm{CD} 25^{\text {high }} \mathrm{CD} 127^{\text {low- }}$ FoxP $3^{+}$, no significant difference was found between the healthy and allergic children regardless of maternal allergy status (Fig. 1A). However, when only two-colour flow cytometry analysis $\left(\mathrm{CD} 4^{+} \mathrm{CD} 25^{+}\right)$was performed, healthy children had significantly increased proportion of these cells $(p=0.0495)$ (Fig. 1B). Proportion of Tregs considered as $\mathrm{CD}^{+}{ }^{+} \mathrm{FoxP}^{+}$was not different in cord blood of healthy and allergic children, Fig. 1C).

After further dividing the two groups of children according to maternal allergy status, there was no difference in $\mathrm{CD} 4{ }^{+} \mathrm{CD} 25^{\text {high }} \mathrm{CD} 127^{\text {low }} \mathrm{FoxP} 3^{+}$Tregs (Fig. 2A). The increase of $\mathrm{CD} 4{ }^{+} \mathrm{CD} 25^{+}$cells in healthy children has
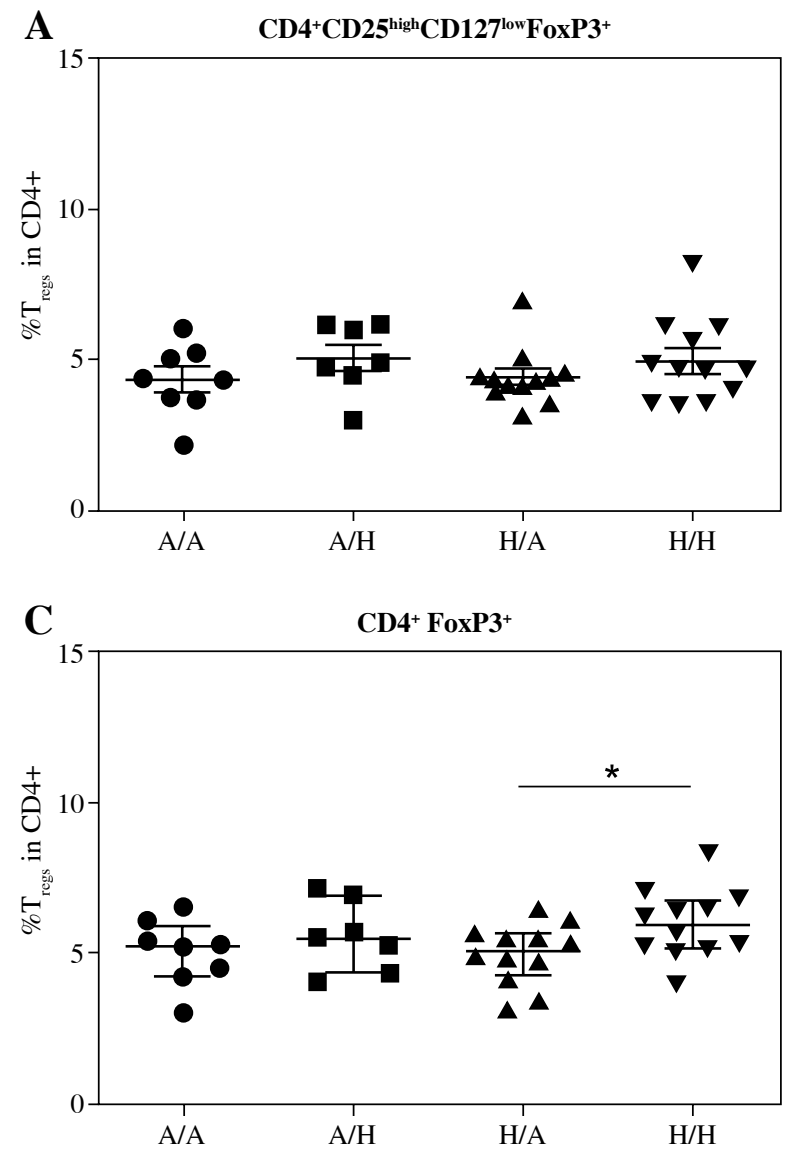

been driven mainly by the group of healthy children of healthy mothers (i.e., from the low-risk group; $\mathrm{H} / \mathrm{H}$ ), who had increased proportion of these cells compared both with allergic children of healthy mothers $(\mathrm{A} / \mathrm{H} ; p=0.0008)$ and with healthy children of allergic mothers $(\mathrm{H} / \mathrm{A} ; p=0.0418)$ (Fig. 2B). Notably, in the group of children of allergic mothers (i.e., the high-risk group) no trend towards lower population of $\mathrm{CD}^{+} \mathrm{CD} 25^{+}$cells in allergic children is discernible, possibly hinting at a different composition or role of this population between the low-risk and high-risk groups. In addition to that, comparison of Tregs based on positivity for CD4 and FoxP3 revealed significantly increased proportion of $\mathrm{CD}^{+}{ }^{+} \mathrm{FoxP} 3^{+}$Tregs in the group of healthy children of healthy mothers in comparison to allergic children of healthy mothers $(p=0.0392)$ (Fig. 2C).

As the observed trends in Treg proportions were somewhat inconsistent with the original findings and other studies published previously $[17,25]$ and our current cohort represented a smaller subset of the subjects included in the original study, we decided to confirm whether the originally described differences between children of allergic and healthy mothers are detectable in our current data. First, we compared Treg proportions between the two groups. While difference in the proportion of $\mathrm{CD} 4{ }^{+} \mathrm{CD} 25^{\text {high }} \mathrm{CD} 127^{\text {low }} \mathrm{FoxP} 3^{+}$ does not reach statistical significance, there is a noticeable

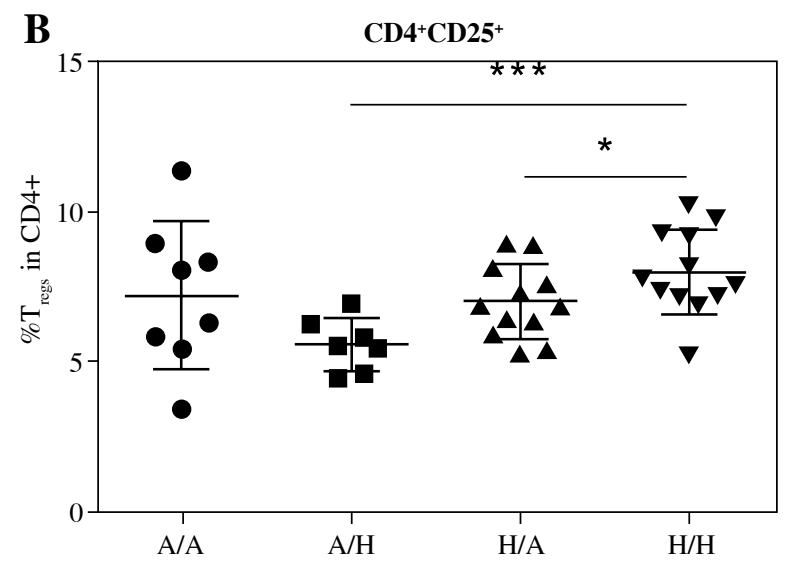

Fig. 2. Proportions of regulatory T cells (Tregs) in cord blood evaluated according to the children's allergy status and maternal allergy status. Samples of cord blood of allergic children of allergic mothers (A/A, $n=8)$, allergic children of healthy mothers $(\mathrm{A} / \mathrm{H}, n=7)$, healthy children of allergic mothers $(\mathrm{H} / \mathrm{A}, n=12)$ and healthy children of healthy mothers $(\mathrm{H} / \mathrm{H}, n=12)$ were stained and analysed by flow cytometry. A) Four-color cytometry analysis of $\mathrm{CD} 4{ }^{+} \mathrm{CD} 25^{\text {high }} \mathrm{CD} 127^{\text {low }} \mathrm{FoxP} 3^{+}$Tregs; B) Two-color cytometry analysis of $\mathrm{CD} 4{ }^{+} \mathrm{CD} 25^{+}$cells; C) Twocolor cytometry analysis of $\mathrm{CD}^{+}{ }^{+} \mathrm{FoxP}^{+}{ }^{+}$Tregs; $* p \leq 0.05$; $* * * p \leq 0.001$ 
trend towards higher numbers of these cells in children of healthy mothers $(\mathrm{p}=0.0559)$ (Supplementary Fig. 2A). The proportion of $\mathrm{CD} 4{ }^{+} \mathrm{CD} 25^{+}$subpopulation is comparable in both groups (Supplementary Fig. 2B). Similarly, no significant difference was observed between groups of children of healthy and allergic mothers in Tregs characterised as $\mathrm{CD}^{+}{ }^{+} \mathrm{FoxP}^{+}$cells (Supplementary Fig. 2C).

\section{MFI of FoxP3}

The original study included the MFI of FoxP3 as one of markers characterising Treg function [14, 17]. After analysing the data divided according to the child's allergy status, we found no difference in MFI of FoxP3 between the two basic groups (healthy children versus allergic children) (Fig. 3A). Further subdivision according to the allergy status of the mother likewise revealed no difference among the groups (Fig. 3B), implying that MFI of FoxP3 may not associate with the risk of allergy development strongly enough to serve as a useful predictive factor.

\section{Intracellular regulatory cytokines IL-10 and TGF- $\beta$}

Further, we investigated whether there was lower intracellular presence of the major regulatory cytokines IL-10 and TGF- $\beta$ in Treg of cord blood of children who developed allergy. No difference was found between the two basic groups for either IL-10 (Fig. 4A) or TGF- $\beta$ (Fig. 4B), although a tendency toward higher values can be discerned in the healthy children. Upon further division of the samples based on the allergy status of the mothers, we uncovered significantly higher proportion of IL-10 ${ }^{+}$ Tregs in cord blood of healthy children of healthy mothers $(\mathrm{H} / \mathrm{H})$ compared with both groups of high-risk children:

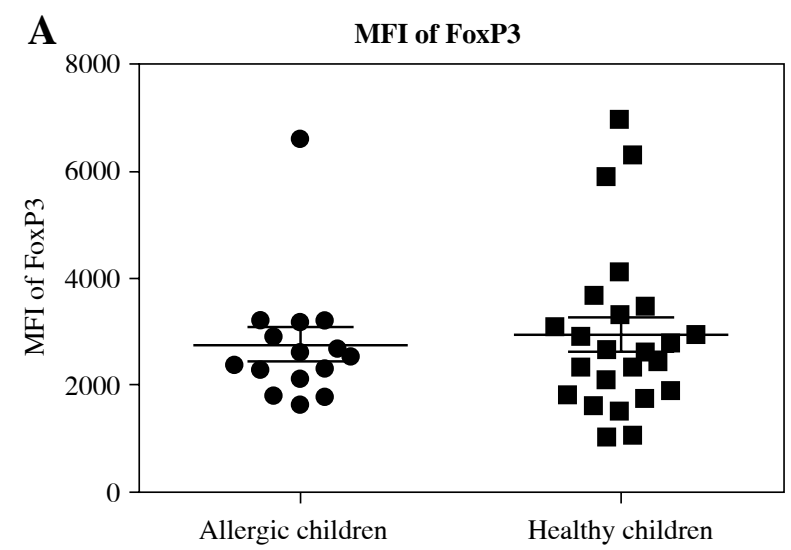

healthy children of allergic mothers $(\mathrm{H} / \mathrm{A} ; p=0.0146)$ and allergic children of allergic mothers (A/A; $p=0.0306)$ (Fig. 5A). Furthermore, lower proportion of TGF- $\beta^{+}$Tregs was observed in allergic children of allergic mothers (A/A) compared with all the other groups, i.e. allergic children of healthy mothers $(\mathrm{A} / \mathrm{H} ; p=0.0205)$, healthy children of allergic mothers $(\mathrm{H} / \mathrm{A} ; p=0.0311)$ and healthy children of healthy mothers $(\mathrm{H} / \mathrm{H} ; p=0.02)$ (Fig. 5B). These findings are consistent with the functional importance of these cytokines in allergy and support the notion that their decreased intracellular presence is an important factor for the actual development of allergy, especially within the high-risk group of children of allergic mothers.

To see if the trends of the markers of Treg function described in the original study can be confirmed in the current smaller subgroup of subjects, we compared MFI of FoxP3 and intracellular presence of IL- 10 and TGF- $\beta$ in Tregs from cord blood of children of allergic mothers and children of healthy mothers, regardless of the allergy status of the children themselves. While MFI of FoxP3 did not differ between the groups (Supplementary Fig. 3A), the proportions of both IL- $10^{+}$and TGF- $\beta^{+}$Tregs were significantly lower in the group of children of allergic mothers, regardless of their own allergy status $(p=0.0173$ and 0.0444, respectively) (Supplementary Fig. 3B and 3C, respectively), consistent with the results seen in the larger cohort [17].

Lastly, concentration of cytokines with regulatory functions (IL-10, TGF- $\beta$ ) was determined in cord blood sera of newborns of healthy and allergic mothers. Levels of IL-10 in cord blood sera of healthy children was not significantly increased in comparison to concentration of IL-10 in cord blood sera of children suffering from allergic diseases. There is only non-significant trend to increased values $(p=0.0681)$ (Fig. 6A). After division of children

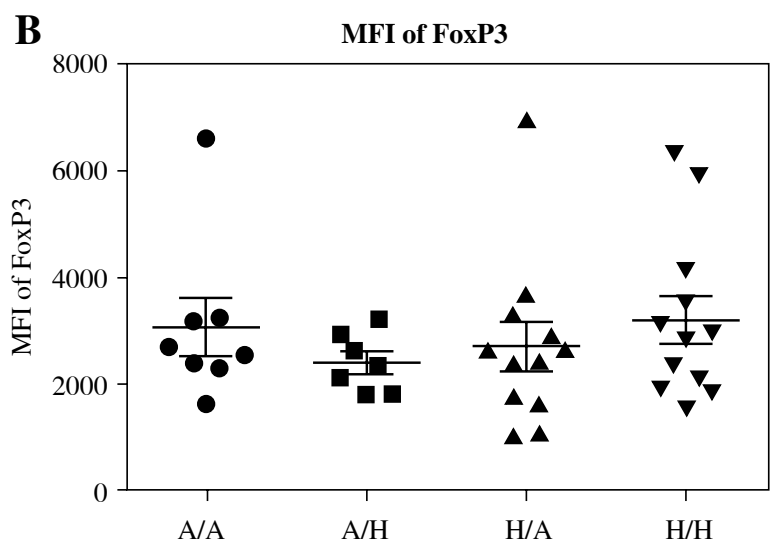

Fig. 3. Median of fluorescence intensity in regulatory T cells (Tregs) in cord blood. Cord blood samples were stained and analysed by flow cytometry. A) Median of fluorescence intensity (MFI) of FoxP3 comparison in cord blood of allergic children $(n=15)$ and healthy children $(n=24)$; B) MFI of FoxP3 comparison in cord blood of allergic children of allergic mothers (A/A, $n=8)$, allergic children of healthy mothers $(\mathrm{A} / \mathrm{H}, n=7)$, healthy children of allergic mothers $(\mathrm{H} / \mathrm{A}, n=12)$ and healthy children of healthy mothers $(\mathrm{H} / \mathrm{H}, n=12)$ 
A

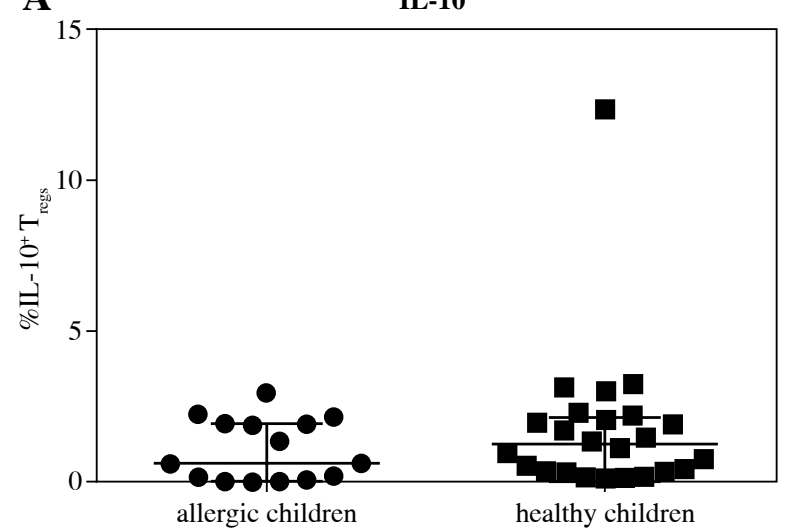

B

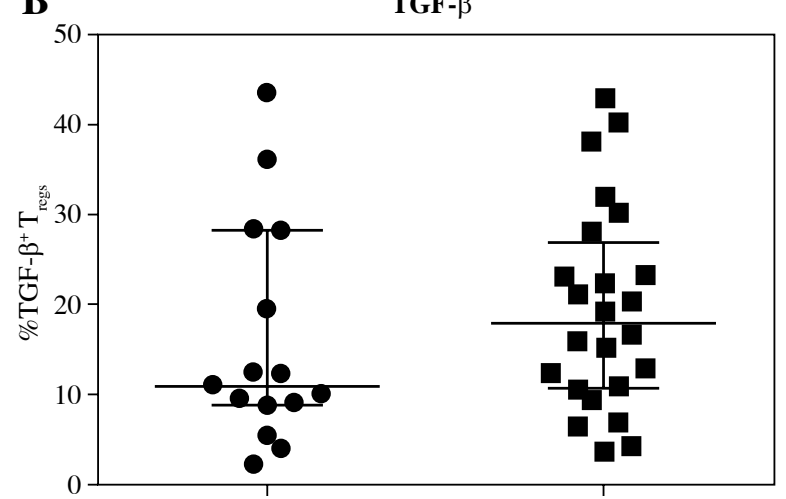

allergic children

healthy children

Fig. 4. Intracellular presence of regulatory cytokines IL-10 and TGF- $\beta$ in regulatory T cells (Tregs) in cord blood evaluated according to the children's allergy status. Samples of cord blood of allergic children $(n=15)$ and healthy children $(n=24)$ were stained and analysed by flow cytometry. A) Percentage of $\mathrm{IL}-10^{+}$Tregs $\left(\mathrm{CD} 4{ }^{+} \mathrm{CD} 25^{\text {high }} \mathrm{CD} 127^{\text {low }}\right)$; B) Percentage of TGF- $\beta^{+}$Tregs $\left(\mathrm{CD} 4^{+} \mathrm{CD} 25^{\text {high }} \mathrm{CD} 127^{\text {low }}\right)$

according to their allergy status and allergic status of their mothers, only significantly higher levels of IL-10 were observed in cord blood sera of healthy children of healthy mothers in comparison to allergic children of allergic mothers $(p=0.0422)$ (Fig. 6B). No difference was found in concentration of TGF- $\beta$ in cord blood sera of healthy and allergic children (Fig. 6C). After subdivision of children according to both their and maternal allergy status, elevated concentration of TGF- $\beta$ was found in cord blood sera of healthy children of healthy mothers in comparison to allergic children of allergy mothers $(p=0.015)$ and healthy children of allergic mothers $(p=0.0402)$ (Fig. 6D).

A

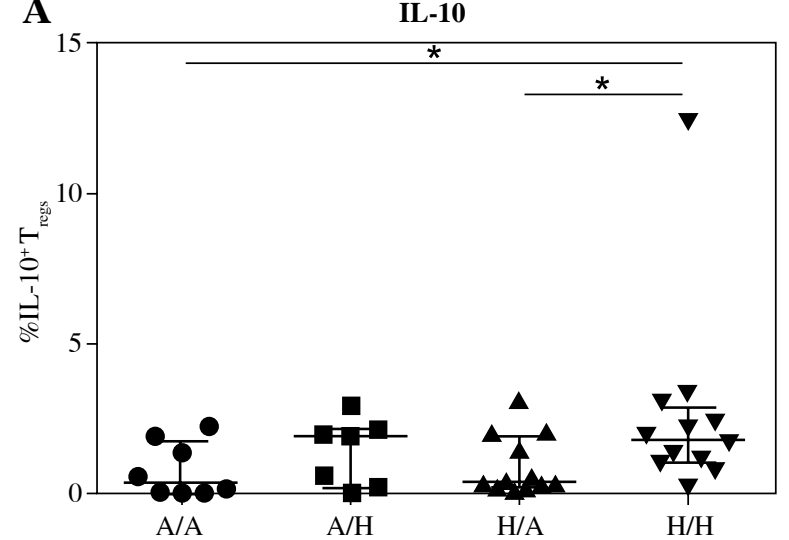

\section{Discussion}

It is well documented that Tregs are critically responsible for immune system regulation, evidenced by the severe autoimmune, inflammatory and allergic disorders accompanying their deficiency or dysfunction $[8,9,28]$. They play a key role in induction and maintenance of immune tolerance, from prenatal period through the infancy into adulthood [3, 29]. Numerous studies aimed to uncover whether analysis of these cells in cord blood, i.e. at the moment of birth, could be used to predict increased risk of allergy, a notion that can only be confirmed upon correlating these parameters with actual allergy development later in life [17, 21, 22, 24, 25].

\section{B}

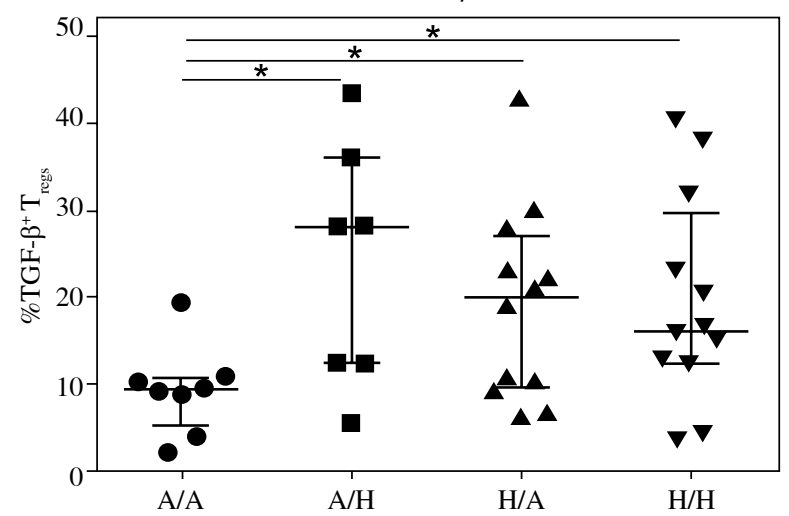

Fig. 5. Intracellular presence of regulatory cytokines IL-10 and TGF- $\beta$ in regulatory T cells (Tregs) in cord blood evaluated according to the children's allergy status and maternal allergy status. Samples of cord blood of allergic children of allergic mothers (A/A, $n=8)$, allergic children of healthy mothers $(\mathrm{A} / \mathrm{H}, n=7)$, healthy children of allergic mothers $(\mathrm{H} / \mathrm{A}, n=12)$ and healthy children of healthy mothers $(\mathrm{H} / \mathrm{H}, n=12)$ were stained and analysed by flow cytometry. A) Percentage of IL- $10^{+}$Tregs $\left(\mathrm{CD} 4{ }^{+} \mathrm{CD} 25^{\text {high }} \mathrm{CD} 127^{\text {low }}\right)$; B $)$ Percentage of TGF- $\beta^{+}$Tregs 
A

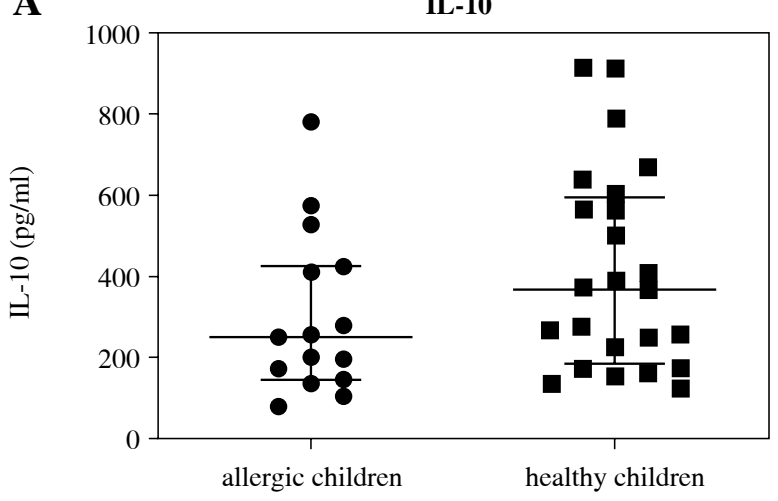

C

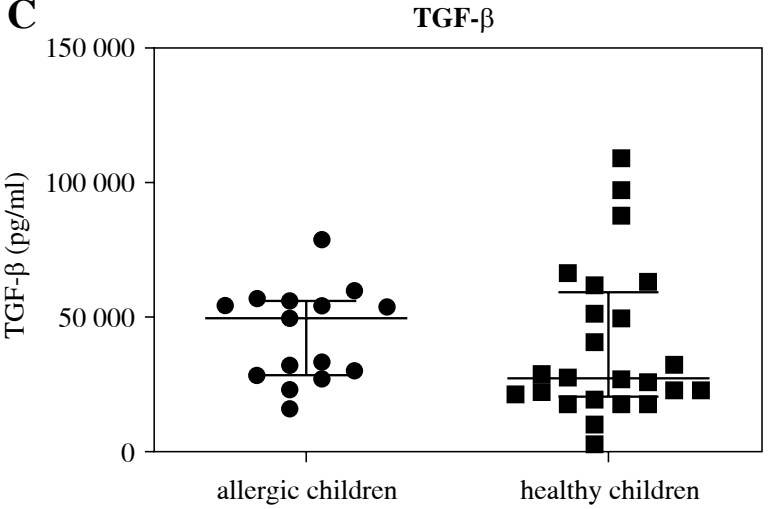

B

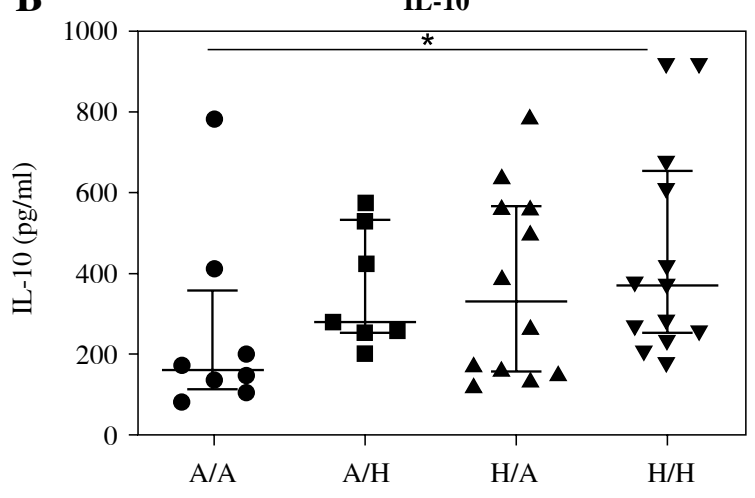

D

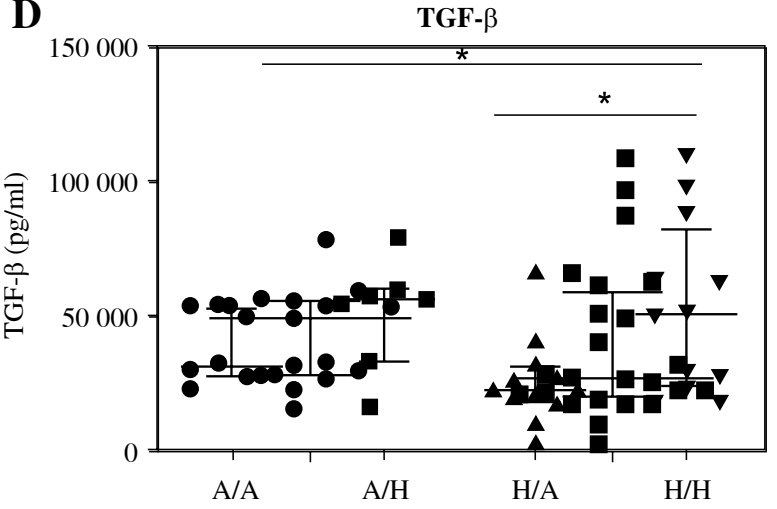

Fig. 6. Cord blood serum levels of regulatory cytokines IL-10 and TGF- $\beta$ evaluated according to the children's allergy status and maternal allergy status. Levels of regulatory cytokines IL-10 and TGF- $\beta$ in cord blood sera were determined using ELISA. A) Concentration of IL-10 in cord blood sera of allergic children $(n=15)$ and healthy children $(n=24)$; B) Concentration of IL-10 in cord blood sera of allergic children of allergic mothers (A/A, $n=8)$, allergic children of healthy mothers ( $\mathrm{A} / \mathrm{H}, n=7)$, healthy children of allergic mothers (H/A, $n=12)$ and healthy children of healthy mothers $(\mathrm{H} / \mathrm{H}, n=12)$; C) Concentration of TGF- $\beta$ in cord blood sera of allergic children $(n=15)$ and healthy children $(n=24)$; D) Concentration of TGF- $\beta$ in cord blood sera of allergic children of allergic mothers (A/A, $n=8$ ), allergic children of healthy mothers ( $\mathrm{A} / \mathrm{H}, n=7)$, healthy children of allergic mothers (H/A, $n=12)$ and healthy children of healthy mothers $(\mathrm{H} / \mathrm{H}, n=12) ; * p \leq 0.05$

In the original study, we measured proportional characteristics and parameters associated with Treg function in samples of cord blood using flow cytometry and analysed the data in the context of allergy development risk according to the allergy status of the mother [17], a commonly accepted predictive marker of increased risk of allergy development [23]. In the current follow-up, we correlated the data with clinical manifestation of allergy in childhood in a subset of the original subjects. One limitation we encountered was the low number of subjects we were able to include for the retrospective analysis - only 39 out of the 153 subjects involved in the previous study. Several factors contributed to the high drop-out rate between the original study and current follow-up. Firstly, a number of questionnaires failed to reach the subjects due to changes in the mailing address. Furthermore, a portion of recipi- ents declined participation in the follow-up study. Finally, several respondents returned incompletely filled questionnaires which failed to provide information necessary for inclusion in analysis. This unfortunately limited the statistical power of our analyses and might account for some of the inconsistencies we observed.

While we did not see any difference in Treg proportions between healthy and allergic children when Tregs were identified as $\mathrm{CD} 4{ }^{+} \mathrm{CD} 25^{\text {high }} \mathrm{CD} 127^{\text {low }} \mathrm{FoxP} 3^{+}$or only $\mathrm{CD}^{+}{ }^{+} \mathrm{FoxP}^{+}$cells, we found out that the population of $\mathrm{CD} 4{ }^{+} \mathrm{CD} 25^{+}$cells was increased in the healthy group. This effect was mainly due to healthy children from the low-risk group, which had higher proportion of these cells than allergic children from the low-risk group as well as healthy children from the high-risk group, with the allergic children from the high-risk group showing notably higher variance. 
Although these results are supported by some published data [24, 26], they are somewhat surprising in the context of the original study, where we found that $\mathrm{CD} 4{ }^{+} \mathrm{CD} 25^{+}$cells were higher in the high-risk group and hypothesised that these cells might in fact be activated CD4 T cells rather than bona fide Tregs [17]. The discrepancy might be attributable to the limited number of subjects available for the retrospective study. Indeed, when the current subjects were stratified according to maternal allergy status without regard to allergy of the children, the original findings could not be confirmed, indicating that results strongly depend on selection of samples within the cohort, as well as the number of subjects analysed.

Further inconsistencies may also be due to inherent properties of Tregs and imperfect identification or understanding of the cells. Regulatory $\mathrm{T}$ cells represent a highly heterogeneous population, which has led to considerably diverse and sometimes contradictory reports. While some groups including ours have previously observed an increase in Tregs in cord blood of children of allergic mothers $[17,25,30]$, in other studies, no difference was found [31] or even the opposite trend was reported [24, 26]. Exacerbating this are confounding factors such as differences in gating strategy and identification of Tregs [17], antibody clones used, especially in the case of FoxP3 [32]; diversity of studied cohorts, particularly considering different genetic background and environment [21, 22]; and methodical inconsistencies, including sample preparation and cohort sizes $[17,33]$.

Moreover, while conventional Tregs are now generally accepted to be $\mathrm{CD} 4{ }^{+} \mathrm{CD} 25^{\text {high }} \mathrm{CD} 127^{\text {low }} \mathrm{FoxP} 3^{+}$cells in humans, numerous other subpopulations have been described in the literature. Expression of transcription factor FoxP3 is considered lineage specific for Tregs and FoxP3 MFI has been linked to suppressive function [14]. However, it has been shown that FoxP3 can be transiently induced by cell activation in non-Treg cells [34] and also that FoxP3- Treg cell populations with suppressive function both exist [35] and play a crucial role in the success of specific allergen immunotherapy $[11,35]$. Currently, epigenetic analysis of demethylation of TSDR in the promotor area of foxp3 gene is proposed to better correspond to Treg phenotype stability and lineage commitment $[6,7]$. Furthermore, while CD25 is the original marker first used to identify Tregs [36], it is also upregulated on activated non-Treg T cells [34], and it has been shown that CD25 ${ }^{\text {high }}$ (approximately the top $2 \%$ of CD25 positivity) better corresponds with bona fide Treg phenotype [37], whereas defining Tregs solely as $\mathrm{CD} 4{ }^{+} \mathrm{CD} 25^{+}$might include activated $\mathrm{T}$ cells.

Considering the diversity of Treg subpopulations, possible contamination by activated $\mathrm{T}$ cells and also marked plasticity of regulatory $\mathrm{T}$ cells $[38,39]$, it is interesting to note that contrary to the situation observed in the groups of low-risk children, the group of children of allergic mothers shows no tendency towards higher proportion of
$\mathrm{CD} 4{ }^{+} \mathrm{CD} 25^{+}$cells in the healthy children. This can possibly be due to the higher variance seen among the allergic subjects from this group. It is perhaps conceivable that the ratios of particular Treg subpopulations within and/or functional significance of the observed $\mathrm{CD} 4{ }^{+} \mathrm{CD} 25^{+}$population might differ between the low-risk and the high-risk groups as defined by maternal allergy status, similar to how some studies report different results based on the inherent risk due to environmental factors (e.g. urban vs. farming environment [21, 22]). Utilising alternative methods of Treg characterisation (such as epigenetic analysis of TSDR) or adding more cell markers associated with Tregs into the panel (e.g. CTLA-4, PD-1, LAG3, CD39 and others [12]) in future studies to better characterise the observed populations would be necessary to conclusively confirm or deny this possibility.

In light of the abovementioned limitations and confounding factors, our findings suggest that simple analysis of proportion of Tregs might not be reliable enough to give a representative picture of perinatal immune status. Functional studies and markers associated with Treg function seem to provide better insight. While in our study there was no significant difference in MFI of FoxP3 between the healthy and allergic children, both IL- $10^{+}$and TGF- $\beta^{+}$ Tregs have been higher in healthy children. Specifically, intracellular presence of IL-10 in Tregs was significantly higher in healthy children of healthy mothers compared with both groups of children of allergic mothers and intracellular presence of TGF- $\beta$ was significantly lower in allergic children of allergic mothers than in all other groups. Taken together with the originally described increased intracellular presence of these cytokines in Tregs in cord blood of children of healthy mothers, the current results indicate that lower IL-10 and TGF- $\beta$ intracellular presence in Tregs correlates with future allergy development, particularly in the high-risk group of children of allergic mothers. This observation suggests that lower levels of regulatory cytokines could be one of the promising predictive markers indicating increased risk of allergy development, which is in accordance with previously published data $[19,20]$.

Our findings are also consistent with the well-established role of these cytokines in immune regulation, including inducing and maintaining tolerance to environmental antigens and control of allergy [10]. IL-10 is the primary effector molecule involved in contact-independent suppression by Tregs [12] and plays an indispensable role in the success of allergen-specific immunotherapy [11, 40]. TGF- $\beta$ plays a vital role in the context of mucosal immunity, including establishment and maintenance of peripheral tolerance towards microbial antigens as well as allergens [41, 42], inducing Tregs in the periphery [42] and generally tuning the mucosal immune system toward tolerogenic conditions [43, 44].

Collectively, our data support the hypothesis that evaluation of early postnatal Treg function, such as intracellular 
presence of regulatory cytokines, might be more useful in predicting allergy development than simple analyses of Treg population proportions, warranting further research using direct functional assays, similar to studies already published $[24,30]$. It might also benefit future studies to include other markers associated with Treg function, such as surface molecules CTLA-4 or PD-1 [45-48], as some studies have already done [30]. Size of the studied cohorts is also a common limiting factor which together with high heterogeneity of allergic diseases may contribute to the inconsistencies reported in literature $[17,33]$. Furthermore, reliable identification of stable Treg phenotype using novel techniques, chiefly epigenetic analysis of TSDR demethylation status, might shed some light into the conflicting reports regarding population proportions of Tregs and their relevance between different subject groups at higher/lower risk of allergy development. Of course, the final verdict regarding the real benefit of the proposed approaches for early prediction of allergy will only be possible upon correlating the data measured at the time of birth with the actual development of allergy later in life. So far, while there is a multitude of studies which characterise Treg populations and functional parameters in cord blood, relatively few studies correlate their findings with clinical data obtained later during life [24].

\section{Acknowledgements}

This research was funded by Czech Health Research Council of the Czech Republic (AZV CR 15-26877A) and research program of Charles University Progres Q25/LF1.

\section{The authors declare no conflict of interest.}

\section{References}

1. Sykes L, MacIntyre DA, Yap XJ, et al. (2012): The Th1:th2 dichotomy of pregnancy and preterm labour. Mediators Inflamm 2012: 967629.

2. Mold JE, Michaëlsson J, Burt TD, et al. (2008): Maternal alloantigens promote the development of tolerogenic fetal regulatory T cells in utero. Science 322: 1562-1565.

3. Jutel M, Akdis CA (2011): T-cell subset regulation in atopy. Curr Allergy Asthma Rep 11: 139-145.

4. Martin R, Nauta AJ, Ben Amor K, et al. (2010): Early life: gut microbiota and immune development in infancy. Benef Microbes 1: 367-382.

5. Strachan DP (1989): Hay fever, hygiene, and household size. BMJ 299: 1259-1260.

6. Kitagawa Y, Ohkura N, Sakaguchi S (2015): Epigenetic control of thymic Treg-cell development. Eur J Immunol 45: 11-16.

7. Iizuka-Koga M, Nakatsukasa H, Ito M, et al. (2017): Induction and maintenance of regulatory $\mathrm{T}$ cells by transcription factors and epigenetic modifications. J Autoimmun 83: 113-121.

8. Bacchetta R, Passerini L, Gambineri E, et al. (2006): Defective regulatory and effector $\mathrm{T}$ cell functions in patients with FOXP3 mutations. J Clin Invest 116: 1713-1722.
9. Barzaghi F, Passerini L, Gambineri E, et al. (2012): Demethylation analysis of the FOXP3 locus shows quantitative defects of regulatory T cells in IPEX-like syndrome. J Autoimmun 38: 49-58.

10. Palomares O, Martín-Fontecha M, Lauener R, et al. (2014): Regulatory $\mathrm{T}$ cells and immune regulation of allergic diseases: roles of IL-10 and TGF- $\beta$. Genes Immun 15: 511-520.

11. Akdis CA, Akdis M (2011): Mechanisms of allergen-specific immunotherapy. J Allergy Clin Immunol 127: 18-27; quiz 28-29.

12. Shevach EM (2009): Mechanisms of Foxp3+ T regulatory cell-mediated suppression. Immunity 30: 636-645.

13. Akdis M, Burgler S, Crameri R, et al. (2011): Interleukins, from 1 to 37, and interferon- $\gamma$ : receptors, functions, and roles in diseases. J Allergy Clin Immunol 127: 701-721.e1-70.

14. Steinborn A, Engst M, Haensch GM, et al. (2010): Small for gestational age (SGA) neonates show reduced suppressive activity of their regulatory T cells. Clin Immunol 134: 188-197.

15. Hrdý J, Zanvit P, Novotná O, et al. (2010): Cytokine expression in cord blood cells of children of healthy and allergic mothers. Folia Microbiol (Praha) 55: 515-519.

16. Hrdý J, Vlasáková K, Černý V, et al. (2018): Decreased allergy incidence in children supplemented with E. coli O83: K24:H31 and its possible modes of action. Eur J Immunol 48: 2015-2030.

17. Hrdý J, Kocourková I, Prokešová L (2012): Impaired function of regulatory $\mathrm{T}$ cells in cord blood of children of allergic mothers. Clin Exp Immunol 170: 10-17.

18. Peters JL, Cohen S, Staudenmayer J, et al. (2012): Prenatal negative life events increases cord blood IgE: interactions with dust mite allergen and maternal atopy. Allergy 67: 545-551.

19. Belderbos ME, Knol EF, Houben ML, et al. (2012): Low neonatal Toll-like receptor 4-mediated interleukin-10 production is associated with subsequent atopic dermatitis. Clin Exp Allergy 42: 66-75.

20. Bullens DMA, Kasran A, Dilissen E, Ceuppens JL (2012): Neonatal IL-10 production and risk of allergy development. Clin Exp Allergy 42: 483-484.

21. Rindsjö E, Joerink M, Johansson C, et al. (2010): Maternal allergic disease does not affect the phenotype of $\mathrm{T}$ and $\mathrm{B}$ cells or the immune response to allergens in neonates: no effect of maternal allergy on neonatal lymphocytes. Allergy 65: 822-830.

22. Schaub B, Liu J, Höppler S, et al. (2009): Maternal farm exposure modulates neonatal immune mechanisms through regulatory T cells. J Allergy Clin Immunol 123: 774-782.e5.

23. Prescott SL, King B, Strong TL, Holt PG (2003): The value of perinatal immune responses in predicting allergic disease at 6 years of age. Allergy 58: 1187-1194.

24. Meng SS, Gao R, Yan BD, et al. (2016): Maternal allergic disease history affects childhood allergy development through impairment of neonatal regulatory T-cells. Respir Res 17: 114.

25. Strömbeck A, Rabe H, Lundell AC, et al. (2014): High proportions of FOXP3 + CD25 high T cells in neonates are positively associated with allergic sensitization later in childhood. Clin Exp Allergy 44: 940-952.

26. Hinz D, Bauer M, Röder S, et al. (2012): Cord blood Tregs with stable FOXP3 expression are influenced by prenatal environment and associated with atopic dermatitis at the age of one year. Allergy 67: 380-389. 
27. Bullens DMA, Seys S, Kasran A, et al. (2015): Low cord blood Foxp3/CD3 $\gamma$ mRNA ratios: a marker of increased risk for allergy development. Clin Exp Allergy 45: 232-237.

28. Pedros C, Duguet F, Saoudi A, Chabod M (2016): Disrupted regulatory $\mathrm{T}$ cell homeostasis in inflammatory bowel diseases. World J Gastroenterol 22: 974-995.

29. Jones CA, Holloway JA, Warner JO (2000): Does atopic disease start in foetal life? Allergy 55: 2-10.

30. Černý V, Hrdý J, Novotná O, et al. (2018): Distinct characteristics of Tregs of newborns of healthy and allergic mothers. PLoS One 13: e0207998.

31. Fu Y, Lou H, Wang Ch, et al. (2013): T cell subsets in cord blood are influenced by maternal allergy and associated with atopic dermatitis. Pediatr Allergy Immunol 24: 178-186.

32. Law JP, Hirschkorn DF, Owen RE, et al. (2009): The importance of Foxp3 antibody and fixation/permeabilization buffer combinations in identifying CD4+CD25+Foxp3+ regulatory T cells. Cytometry A 75: 1040-1050.

33. Smith M, Tourigny MR, Noakes P, et al. (2008): Children with egg allergy have evidence of reduced neonatal CD4(+) $\mathrm{CD} 25(+) \mathrm{CD} 127(\mathrm{lo} /-)$ regulatory T cell function. J Allergy Clin Immunol 121: 1460-1466, 1466.e1-7.

34. Kmieciak M, Gowda M, Graham L, et al. (2009): Human $\mathrm{T}$ cells express CD25 and Foxp3 upon activation and exhibit effector/memory phenotypes without any regulatory/suppressor function. J Transl Med 7: 89.

35. Akdis CA, Akdis M (2015): Mechanisms of allergen-specific immunotherapy and immune tolerance to allergens. World Allergy Organ J 8: 17.

36. Sakaguchi S, Sakaguchi N, Asano M, et al. (1995): Immunologic self-tolerance maintained by activated $\mathrm{T}$ cells expressing IL-2 receptor alpha-chains (CD25). Breakdown of a single mechanism of self-tolerance causes various autoimmune diseases. J Immunol 155: 1151-1164.

37. Roncador G, Brown PJ, Maestre L, et al. (2005): Analysis of FOXP3 protein expression in human $\mathrm{CD} 4+\mathrm{CD} 25+$ regulatory $\mathrm{T}$ cells at the single-cell level. Eur J Immunol 35: 1681-1691.

38. Sakaguchi S, Vignali DAA, Rudensky AY, et al. (2013): The plasticity and stability of regulatory T cells. Nat Rev Immunol 13: 461-467.

39. Barnaba V, Schinzari V (2013): Induction, control, and plasticity of Treg cells: the immune regulatory network revised? Eur J Immunol 43: 318-322.

40. Akdis CA, Akdis M (2015): Mechanisms of allergen-specific immunotherapy and immune tolerance to allergens. World Allergy Organ J 8: 1-12.

41. Edwards JP, Hand TW, da Fonseca DM, et al. (2016): The GARP/Latent TGF- $\beta 1$ complex on Treg cells modulates the induction of peripherally derived Treg cells during oral tolerance. Eur J Immunol 46: 1480-1489.

42. Josefowicz SZ, Niec RE, Kim HY, et al. (2012): Extrathymically generated regulatory $\mathrm{T}$ cells control mucosal TH2 inflammation. Nature 482: 395-399.

43. Konkel JE, Chen W (2011): Balancing acts: the role of TGF- $\beta$ in the mucosal immune system. Trends Mol Med 17: 668-676.

44. Konkel JE, Zhang D, Zanvit P, et al. (2017): Transforming growth factor- $\beta$ signaling in regulatory $\mathrm{T}$ cells controls $\mathrm{T}$ helper-17 cells and tissue-specific immune responses. Immunity 46: 660-674.

45. Kumar S, Verma AK, Das M, Dwivedi PD (2013): A molecular insight of CTLA-4 in food allergy. Immunol Lett 149: 101-109.
46. Munthe-Kaas MC, Carlsen KH, et al. (2004): CTLA-4 polymorphisms in allergy and asthma and the TH1/ TH2 paradigm. J Allergy Clin Immunol 114: 280-287.

47. McAlees JW, Lajoie S, Dienger K, et al. (2015): Differential control of CD4 + T-cell subsets by the PD-1/PD-L1 axis in a mouse model of allergic asthma: cellular immune response. Eur J Immunol 45: 1019-1029.

48. McGee HS, Yagita H, Shao Z, Agrawal DK (2010): Programmed death-1 antibody blocks therapeutic effects of T-regulatory cells in cockroach antigen-induced allergic asthma. Am J Respir Cell Mol Biol 43: 432-442. 